\title{
A Study on the Female Chest Contour with Elliptic Fourier Analysis *
}

\author{
Hui-Yao Zhang ${ }^{\mathrm{a}}$, Duan $\mathrm{Li}^{\mathrm{a}}$, Hao-Yang Xie ${ }^{\mathrm{a}}$, Yue-Qi Zhonga,b,* \\ ${ }^{a}$ College of Textiles, Donghua University, Shanghai 201620, China \\ ${ }^{\mathrm{b}}$ Key Lab of Textile Science and Technology, Ministry of Education, Shanghai 201620, China
}

\begin{abstract}
Elliptic Fourier Analysis(EFA) is an important tool in shape reconstruction. To get the best female subject fitting result, we took the following processes. Firstly, three kinds of sampling methods were taken, including curvature sampling, random sampling and equally spaced sampling. According to our experiment result, equally spaced had the best performance. Secondly, eclectic sampling density was decided by the mean distance and conference. Finally, the number of harmonics used by EFA was decided by the experimental error requirement. The experimental results indicate that the EFA was an powerful and efficient approach in chest shape fitting.
\end{abstract}

Keywords: Elliptic Fourier Analysis; Sampling Method; Sampling Points; Harmonics

\section{Introduction}

In 1982, Kuhl [1] used Elliptic Fourier as the main method to describe object silhouette. In other related field, Elliptic Fourier has been taken to describe contour curve and Elliptic Fourier Descriptors (EFD) was treated as an important tool to calculate differences [2-4] between different object curve. For example, Neto J C used EFDs to describe and analyze the shape of plant leaf, and the coefficient of EFDs of plant leaf was taken to identify the type of plant. Martin Friess [5] used Elliptic Fourier to study the form of three dimensional curves and extract characteristic curves from scanned human body.

Human chest size are very important factor for a suitable cloth [6-8], a costume designer designs a virtual [9] or real cloth.

In this paper, EFA was employed as the core approach to implement the whole study. Based on advice from tailors, A facile hypothesis was proposed that the number of sampling points is based on chest girth and maximum distance error and mean error range is $1 \mathrm{~mm}$ and $0.5 \mathrm{~mm}$, respectively. Different sampling method leads to different fitting result. As a result, with different

${ }^{\star}$ Project supported by National Natural Science Foundation of China (No. 61572124) and Shanghai Natural Science Foundation (No. 14ZR1401100).

* Corresponding author.

Email address: zhyq@dhu.edu.cn (Yue-Qi Zhong). 
sampling method, sampling points and harmonics, different approach was taken to reconstruct the fitting curve. At last, sixteen individuals were used to verify the accurate of our fitting.

\section{Methodology}

\subsection{Data Collection}

First, the chest circumference was obtained by manual measurement. Then the Kinnect was used to scan a female bust. After the human point cloud data was collected, the OBJ file was imported into GeoMagic Studio 12 [10]. According to the feature points was chosen manually before scanning, the model was cut horizontally and the chest curve was obtained. Last, several different curve was created with various sampling methods.

\section{$2.2 \quad$ Elliptic Fourier Analysis}

The coefficients of the Fourier Series were referred to as "Elliptic Fourier Descriptors" (EFDs) [6], which could be implemented to reconstruct the original shape with major features. EFDs was first proposed by [11], it provided robustness to translation, rotation and scale if the EFDs were normalized [12]. Lots of studies used EFDs to research, for example, the characteristics of animals and plants [13-16], anthropology [17], hand-written recognition [18] and aircraft contour description [19].

Suppose there are $K$ discrete sampling points along a closed contour. This contour can be represented by a sequence of $x$ and $y$ coordinates which are ordered by counter-clockwise from an arbitrary starting point. The length from the starting point to the $p$-th point is denoted by $t_{p}$, and the perimeter of the whole contour is denoted by $T$, and $T=t_{K}$ where $K$ is the total number of the discrete sampling points and $\Delta t_{i}$ is the distance between the (i-1)-th point and $i$-th point. For each sampling point, the coordinates of $p$-th along $x$ and $y$ directions are:

$$
\begin{aligned}
& t_{p}=\sum_{i=1}^{p} \Delta t_{i} \\
& x_{p}=\sum_{i=1}^{p} \Delta x_{i} \\
& y_{p}=\sum_{i=1}^{p} \Delta y_{i}
\end{aligned}
$$

where $\Delta x_{i}$ and $\Delta y_{i}$ are the distance from $(i-1)$-th point to the $i$-th point along the $x$ and $y$ axes respectively. Thus, the Elliptic Fourier expansions of $x$ and $y$ coordinate along the contour are

$$
\begin{aligned}
& x_{p}=A_{0}+\sum_{n=1}^{\infty}\left(a_{n} \cos \frac{2 n \pi t_{p}}{T}+b_{n} \sin \frac{2 n \pi t_{p}}{T}\right) \\
& y_{p}=C_{0}+\sum_{n=1}^{\infty}\left(c_{n} \cos \frac{2 n \pi t_{p}}{T}+d_{n} \sin \frac{2 n \pi t_{p}}{T}\right)
\end{aligned}
$$


The coefficients $a_{n}, b_{n}, c_{n}$ and $d_{n}$ actually have the capacity to describe the outline of the original section and are known as Elliptic Fourier coefficient of the $n$-th harmonic. Notice that each harmonic is denoted by four Elliptic Fourier coefficients and the first harmonic is used to align the contour [19].

$$
\begin{aligned}
& a_{n}=\frac{T}{2 n^{2} \pi^{2}} \sum_{p=1}^{K} \frac{\Delta x_{p}}{\Delta t_{p}}\left(\cos \frac{2 n \pi t_{p}}{T}-\cos \frac{2 n \pi t_{p-1}}{T}\right) \\
& b_{n}=\frac{T}{2 n^{2} \pi^{2}} \sum_{p=1}^{K} \frac{\Delta x_{p}}{\Delta t_{p}}\left(\cos \frac{2 n \pi t_{p}}{T}-\sin \frac{2 n \pi t_{p-1}}{T}\right) \\
& c_{n}=\frac{T}{2 n^{2} \pi^{2}} \sum_{p=1}^{K} \frac{\Delta y_{p}}{\Delta t_{p}}\left(\cos \frac{2 n \pi t_{p}}{T}-\cos \frac{2 n \pi t_{p-1}}{T}\right) \\
& d_{n}=\frac{T}{2 n^{2} \pi^{2}} \sum_{p=1}^{K} \frac{\Delta y_{p}}{\Delta t_{p}}\left(\cos \frac{2 n \pi t_{p}}{T}-\sin \frac{2 n \pi t_{p-1}}{T}\right)
\end{aligned}
$$

The equations above are the basic principle and tools were used in this study. First, the Elliptic Fourier coefficient of $N$ harmonics were computed according to sampling points, and then the fitting coordinates were computed based on the original sampling points.

\section{$2.3 \quad$ Error Metrics}

Four parameters were selected to measure the similarity of the original contour and fitting contour: 1) perimeter accuracy, 2) mean distance between original sampling points and fitting points, 3) standard deviation of distance between original sampling points and fitting points and 4) maximum distance between sampling points and reconstruction points.

1) Perimeter was treated as one of the error metrics due to the number of sampling point is closely related to the contour perimeter, furthermore the chest circumference is also a significant factor in garment industry.

Taking $O$ as original/actual contour and $F$ denoted as fitting contour. $P(O)$ and $P(F)$ is the perimeters of the original contour and the fitting contour, respectively. Then perimeter accuracy is computed as

$$
A=\frac{P(F)}{P(O)}
$$

2) Mean distance $(\bar{D})$ reflects the deviation extent between the original shape and reconstruction shape. Mean distance is computed by the following formula where $K$ is the total number of the sampling points.

$$
\bar{D}=\frac{1}{K} \sum_{1}^{K} \operatorname{dist}\left(O_{i}, F_{i}\right) \quad i=1,2, \cdots, K
$$

3) The standard deviation of distance between original sampling points and fitting points () reflects the measure variability which is computed as

$$
\sigma_{D}=\operatorname{std}\left(D_{i}\right)
$$


4) The maximum distance between sampling points and fitting points is denoted as

$$
D_{\max }=\operatorname{Max}\left\{\operatorname{dist}\left(O_{i}, F_{i}\right), i=1,2, \cdots, K\right\}
$$

Now the core algorithm and the methods have been illustrated that was used to measure the errors.

\section{Experimental Results and Discussion}

\subsection{Testing Sampling Methods}

Three common sampling methods were included in this study, curvature sampling, random sampling and equally spaced sampling. In this step, all these three kinds of sampling methods was used to obtain sampling points and the number of sampling point is equal. So 600 sampling points were selected per curve with different sampling methods and the number of harmonics is 18. Table 1 lists the result of the test. (All the three discrete closed curve are created by GeoMagic Studio 12).

Table 1: Comparison of three different sampling methods with 600 discrete points and 18 harmonics

\begin{tabular}{lccc}
\hline Error Metrics & Curvature Sampling & Random Sampling & Equally spaced Sampling \\
\hline Mean Distance (mm) & 6.29 & 9.46 & 0.67 \\
Max Distance (mm) & 9.74 & 12.28 & 1.63 \\
Standard Deviation & 21.06 & 32.14 & 0.62 \\
Perimeter Accuracy & $91.08 \%$ & $97.36 \%$ & $99.65 \%$ \\
\hline
\end{tabular}

As shown in Table 1, the equally spaced sampling has the best performance. So in the following sections equally spaced sampling was selected by default. Figure 1 represents the fitting effects of curvature sampling and equally spaced sampling based on 600 sampling points and 18 harmonics.

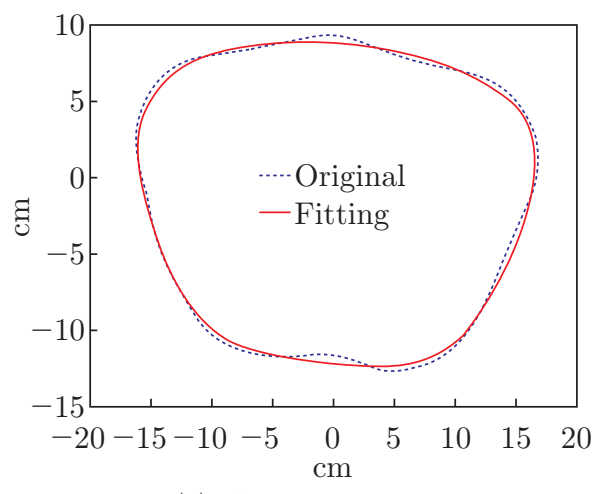

(c) Curvature sampling

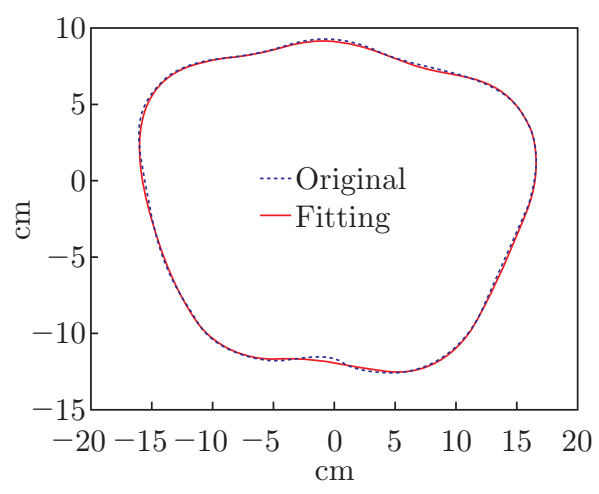

(b) Equally spaced sampling

Fig. 1: The fitting effects of curvature sampling and equally spaced sampling

As shown in Fig. 1, Left is the result of curvature sampling, at the left bottom corner, the gap between two contour was obvious. Right is the result of equally spaced sampling. Both of them use 600 sampling points and 18 harmonics. White is the original contour and red is the fitting contour. 


\subsection{Sampling Density}

As shown in Fig. 2, construction result with 1800 harmonics and only 100 sampling points. The perimeter accuracy is $98.25 \%$. In this step, we decide the best number of sampling points. In Fig. 2, it represents an extreme reconstruction curve in which the sampling points is 120 and the harmonics number is 2000. According to advices from senior tailors and experienced garment designers, $99 \%$ perimeter accuracy, $1 \mathrm{~mm}$ maximum distance and 0.5 mean distance could satisfy practical requirements.

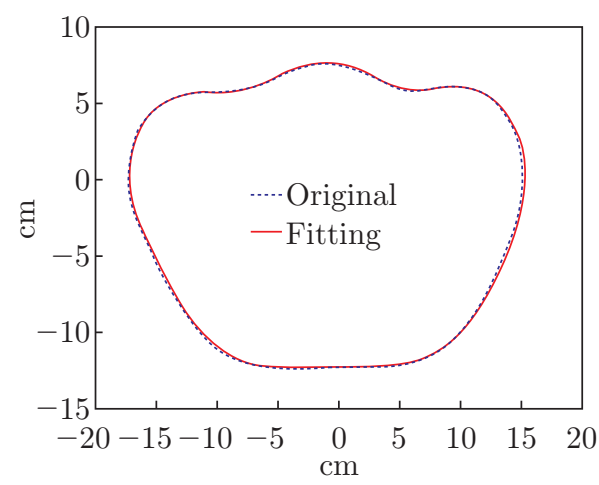

Fig. 2: Construction result with 1800 harmonics and only 100 sampling points

To testify the scanner accuracy, a cylinder was scanned. The diameter if the cylinder is $25 \mathrm{~cm}$ and the height is $80 \mathrm{~cm}$. The scanned cylinder accuracy is analyzed. According to the Fig. 1, the scanning error increased from the center to the both ends, and the scanning error got the maximum value which is less than $0.5 \mathrm{~cm}$, which could satisfy our experiment requirement.

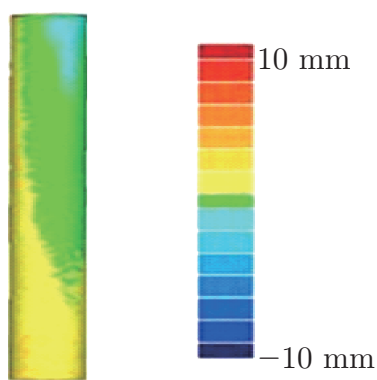

Fig. 3: Testify the scanning system error with a cylinder

\subsection{Reconstruction with Limited Harmonics}

As for the number of harmonics, it is decided based on the fitting result and error metrics. The chest circumference of the following model was $886 \mathrm{~mm}$, so 1772 sampling points was taken based on equal sampling.

With the increase of the harmonics, the Perimeter Accuracy get more accuracy and the value of mean distance, maximum distance and standard deviation become smaller, It can be seen that after the number of sampling points was decided, the only thing need to be done is to test whether the number of harmonics could satisfy the acceptable errors. 

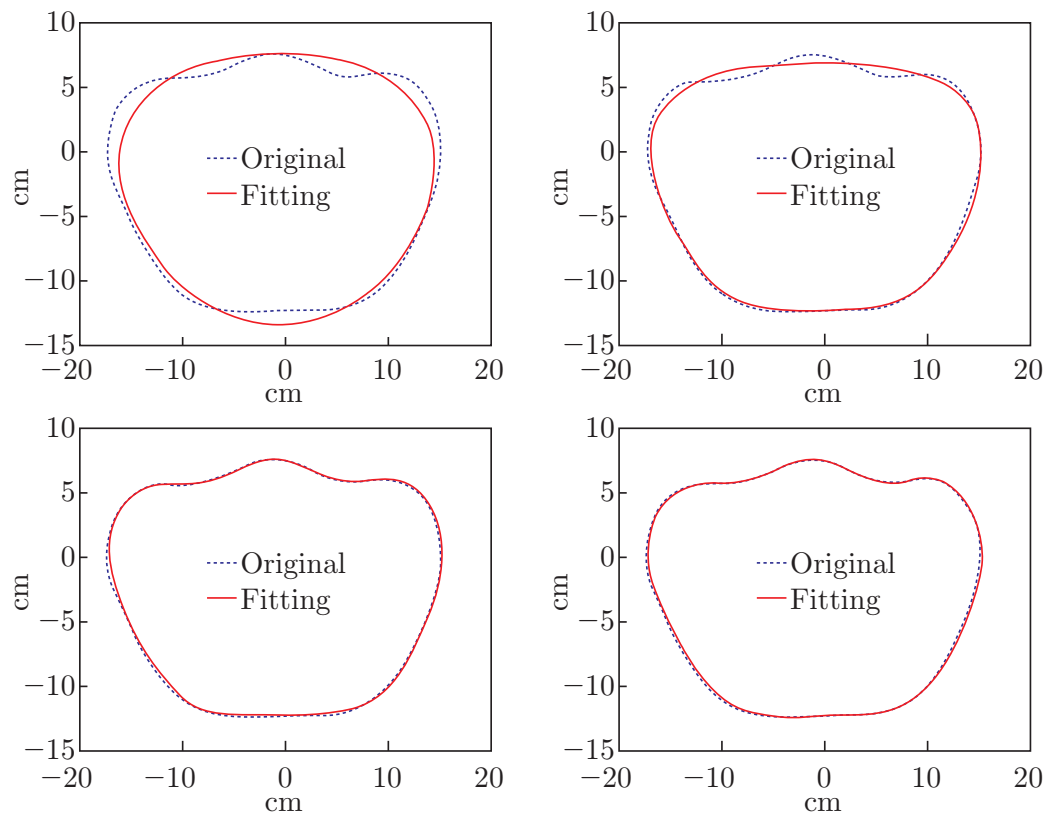

Fig. 4: Four different results with the same 1772 discrete points and different harmonics
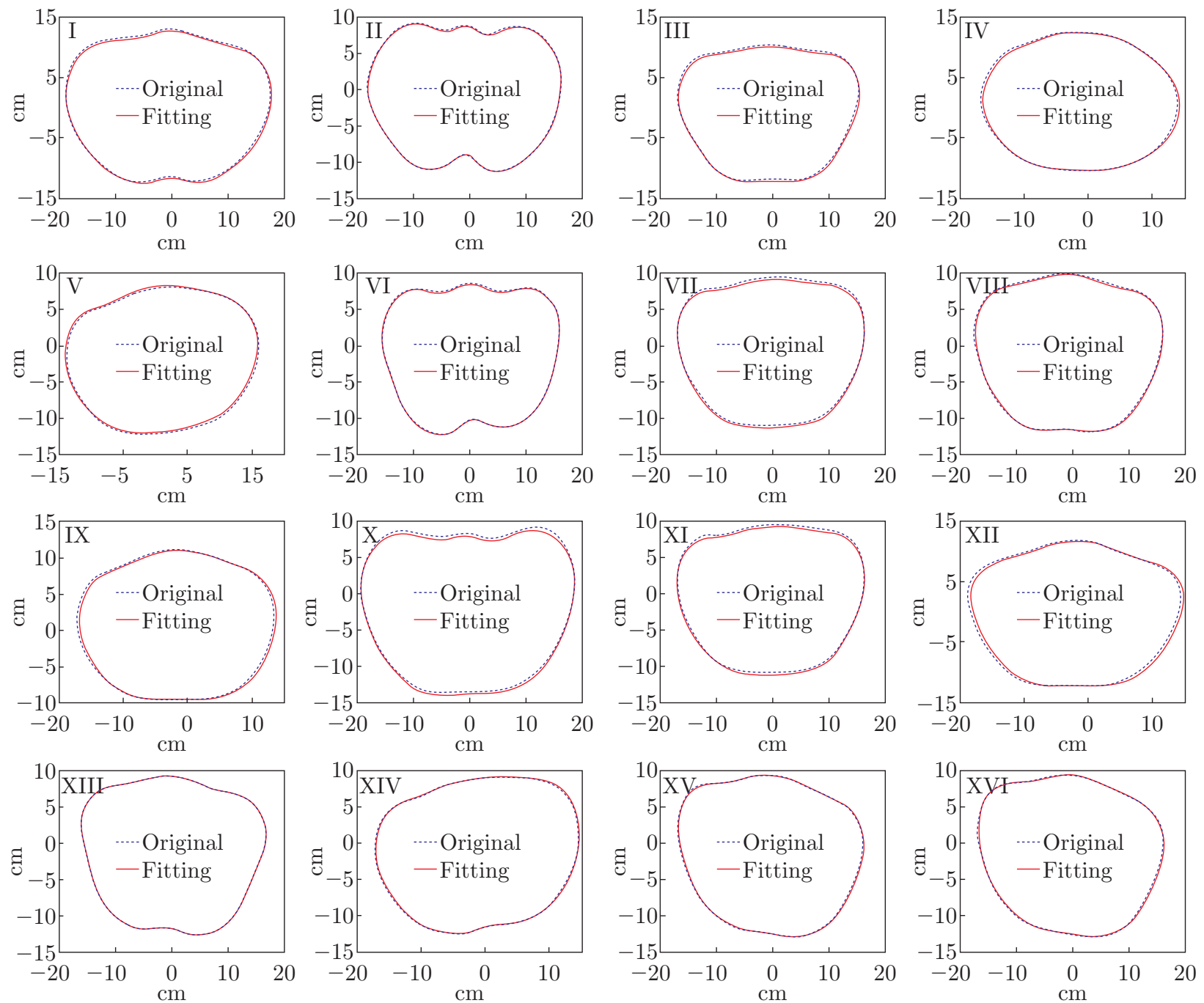

Fig. 5: Construction result of other 16 scanned model. The number of sampling points is based on the girth of chest contour and mean error 
Table 2: Demonstrated the different imitations with the same number of sampling points with equally spaced sampling but different harmonics

\begin{tabular}{ccccc}
\hline Harmonics & Perimeter Accuracy & Mean Distance & Maximum Distance & Standard Deviation \\
\hline 2 & $94.28 \%$ & 6.84 & 12.18 & 4.32 \\
4 & $97.74 \%$ & 2.96 & 7.43 & 2.05 \\
6 & $99.52 \%$ & 1.76 & 2.46 & 0.75 \\
8 & $99.86 \%$ & 0.39 & 0.86 & 0.23 \\
\hline
\end{tabular}

\subsection{Test Validation}

Sixteen female models was used to verify the hypothesis in the study. For all the 16 models, the sum of the sampling points were decided by chest circumference. All of acceptable results can be achieved with limited harmonics in practice. Fig. 5 demonstrates the imitations and Table 3 represents the error metrics.

Table 3: Error metrics of 8 scanned model. The measure unit of perimeter, mean distance and max distance is millimeter

\begin{tabular}{cccccccc}
\hline Model & Perimeter & Sampling Points & Harmonics & Perimeter Accuracy & Mean Distance & Max Distance & Std. \\
\hline I & 790 & 1580 & 21 & $99.84 \%$ & 0.36 & 0.98 & 0.18 \\
II & 926 & 1852 & 23 & $99.79 \%$ & 0.29 & 0.94 & 0.16 \\
III & 792 & 1584 & 18 & $99.82 \%$ & 0.31 & 0.93 & 0.18 \\
IV & 836 & 1672 & 20 & $99.86 \%$ & 0.33 & 0.96 & 0.16 \\
V & 895 & 1790 & 15 & $99.68 \%$ & 0.32 & 0.90 & 0.17 \\
VI & 793 & 1586 & 16 & $99.88 \%$ & 0.34 & 0.97 & 0.16 \\
VII & 862 & 1724 & 21 & $99.71 \%$ & 0.33 & 0.95 & 0.19 \\
VIII & 920 & 1840 & 16 & $99.83 \%$ & 0.29 & 0.98 & 0.17 \\
IX & 824 & 1648 & 18 & $99.81 \%$ & 0.34 & 0.93 & 0.17 \\
X & 914 & 1828 & 20 & $99.84 \%$ & 0.27 & 0.89 & 0.21 \\
XI & 848 & 1696 & 21 & $99.71 \%$ & 0.31 & 0.93 & 0.16 \\
XII & 803 & 1606 & 15 & $99.63 \%$ & 0.28 & 0.97 & 0.19 \\
XIII & 878 & 1756 & 20 & $99.85 \%$ & 0.35 & 0.89 & 0.22 \\
XIV & 908 & 1808 & 18 & $99.87 \%$ & 0.29 & 0.92 & 0.15 \\
XV & 763 & 1526 & 21 & $99.63 \%$ & 0.28 & 0.98 & 0.18 \\
XVI & 875 & 1750 & 20 & $99.88 \%$ & 0.27 & 0.96 & 0.19 \\
\hline
\end{tabular}

\section{Conclusion}

In this paper, EFA was used as the main method to reconstruct female chest contour. Three main variables influence the fitting result. The first is the sampling method, based on our experiment, equally spaced sampling is taken as the best way to obtain sampling point. The second is sampling density, the sampling interval was decided by the mean error and chest circumstance. The third 
factor is harmonics number, the harmonics was decided by the fitting result.

There may be other methods to decide the number of sampling points, however, in practice, the hypothesis we proposed here is effective for the Elliptic Fourier fitting of female chest contour with limited harmonics.

\section{Acknowledgement}

This work is supported by National Natural Science Foundation of China (Grant No. 61572124) and Shanghai Natural Science Foundation (Grant No. 14ZR1401100).

\section{References}

[1] Kuhi FP, Giardina CR. Elliptic Fourier features of a closed contour. COMPUT VISION GRAPH: 1982, 18(3): 236-25.

[2] Adebowale A, Nicholas A, Lamb J, et al. Elliptic Fourier analysis of leaf shape in southern African Strychnos section Densiflorae (Loganiaceae) Bot. J. Linn. Soc.: 2012, 170(4): 542-553.

[3] Neto JC, Meyer G, Jones DD, et al. Plant species identification using Elliptic Fourier leaf shape analysis. Computers and Electronics in Agriculture: 2006, 50(2): 121-134.

[4] Viscosi V, Fortini P. Leaf shape variation and differentiation in three sympatric white oak species revealed by elliptic Fourier analysis. Nordic Journal of Botany: 2011, 29 (5): 632-640.

[5] Friess M, Rohlf FJ, Hsiao HW. Quantitative Assessment of Human Body Shape Using Fourier Analysis CORNER BD, LI P, PARGAS R P. Three-Dimensional Image Capture and Applications Vi. Bellingham: Spie-Int Soc Optical Engineering: 2004, 117-124.

[6] Liu H, Zhang M, Chen DS, et al. Study on bust curve of standard female figure. Journal of Textile Research: 2011, 32(2): 117-120.

[7] Shang XM, Lu C, Wang H. Human body circumference curve fitting model base on automatic measurement. Journal of Textile Research: 2010, 31(4): 128-131, 138.

[8] Abels A, Kruusmaa M. Design of a shape-changing anthropomorphic mannequin for tailoring applications. International Conference on Advanced Robotics. IEEE: 2009, 1-6.

[9] Petrak S, Rogale D. Systematic representation and application of a 3D computer aided garment construction method. Int J Clothing Sci Tech: 2006, 3: 179-187.

[10] $\mathrm{Hu}$ YF. The application of geomagic studio software in reverse engineering post-processing. Manuf Autom: 2009, 5: 501-524.

[11] KUJL. Elliptic Fourier Features of a Closed Contour. Comput Graph Imag: 1982, 18: 236-258.

[12] Bracewell R N. The Fourier transform. Scientific American: 1989, 260: 62-69.

[13] Iwata H, Niikura S, Matsuura S, Takano Y, Ukai Y. Interaction between genetic effects and soil type in diallel analysis of root shape and size of Japanese radish (Raphaunussativus L). Breeding Science: 2004, 54: 313-385.

[14] Tracey SR, Lyle JM, Duhamelb G. Application of elliptical Fourier analysis of otolith form as a tool for stock identification. Fisheries Research: 2006, 77: 138-147.

[15] Rohlf FJ, Archie JW. Acomparison of Fourier methods for the description of wing shape in mosquitoes. Syst. Zool: 1984, 33: 302-317. 
[16] Neto JC, Meyer GE, Jones DD, Samal AK. Plant species identification using elliptic fourier leaf shape analysis. COMPUT ELECTRON AGR: 2006, 50(2): 121-134.

[17] Daegling DJ, Jungers WL. Elliptical Fourier analysis of symphyseal shape in great ape mandibles. Hum. Evol: 2000, 39: 107-122.

[18] Granlund GH. Fourier Preprocessing for Hand Print Character Recognition. Trans Comput: 1972, 21: 195-201.

[19] Wallace TP, Wintz PA. An efficient three-dimensional aircraft recognition algorithm using normalized fourier descriptors. Comput Graph Image: 1980, 13: 99-126. 